\title{
Ultra Wide Band LPDA Design using HFSS
}

\author{
Rakhi Dua, Dr. Arti Vaish \\ Ph.D Student JJTU Jhunjhunu, India \\ Asst. Profassor Galgotia College of Engineering Grater Noida, India
}

\begin{abstract}
This paper describes the development of LPDA using HFSS. Log Periodic Antennas are antennas with high gain, large bandwidth, large front to back ratio and low cost. These antennas are quite convenient for commercial broadcast and radio signal detection. The UWB LPDA antenna is slightly modified to cover $1 \mathrm{GHz}$ to $4 \mathrm{GHz}$ bandwidth. The radiation patterns of sum and differential modes show good gain and null depth in the end-fire direction, respectively. The performance of the designed antenna is verified by finite integration and finite element method comparisons. The design of LPDA is reduced to attain better impedance matching.
\end{abstract}

Keywords: LPDA,HFSS,UWB

\section{INTRODUCTION}

With the recent interest in UWB systems for communication applications there is a surge of interest in UWB antennas. These systems make use of UWB pulse rather than narrow band pulses. LPDA's are convenient for wideband applications. While there have been many novel antenna designs introduced to satisfy UWB antenna criteria, the prevalence, simplicity and familiarity of the log-periodic array would make it an appealing choice if one could suggest a means to overcome phase shift issues with properly centered phases. Because of the frequency dependent phase shifts that exist between the elements of this antenna the log-periodic array is known to be a very dispersive environment for a pulse excitation; and, consequently, its output signal is a severely distorted version of the input pulse. Consequently, this dispersion-engineered log-periodic array may have an important impact on UWB system designs and applications.

\section{LPDA GEOMETRY}

Conventional LPDA is shown in fig.1. A standard crisscross connection was assumed and implemented with a two parallel layer structure that is represented in fig.1.

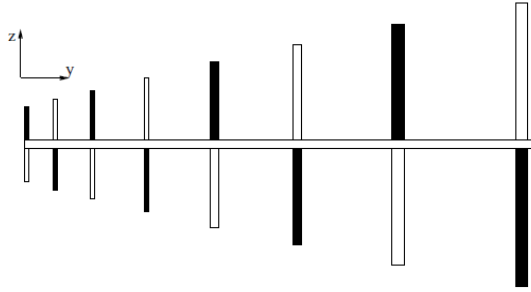

Fig.1.Conventional LPDA

We note that it was found to be necessary to calculate the far field with the indicated combined analyticalnumerical approach because a calculation with HFSS alone was not feasible computationally because of the extremely large memory and time requirements. To overcome this problem a modified LPDA is taken into consideration. A diagram of modified LPDA is shown in fig.2.The simulation of such type of antenna can be done by HFSS. The current magnitude distribution essentially remains the same while current phase is modified.

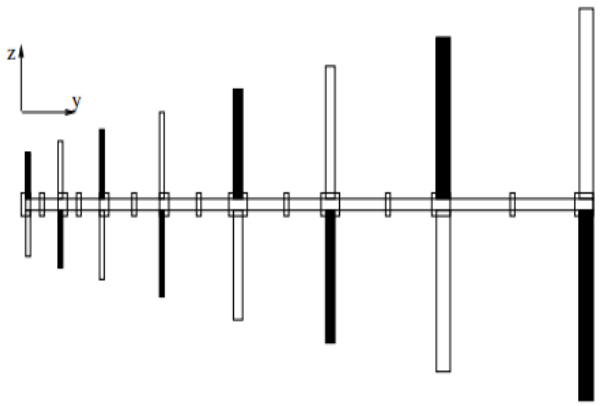

Fig.2.Modified LPDA 
For the purpose of performing frequency domain analysis ANSOFT high frequency structure simulator is required. It should be pointed out that the HFSS simulator solves the curl-curl - k2 form of the electric field equation obtained from Maxwell's equations in the frequency domain with the finite element method.

\section{Simulation RESUlT}

Radiation pattern of conventional and modified LPDA'S are shown in fig.3. The HFSS simulations were performed for an eight element printed dipole LPDA antenna.

The reason that the basic modified LPDA failed to maintain this highly desirable frequency domain antenna pattern property in the low frequency range is that although the current phase was adjusted to the optimum solution value at each of the resonant frequencies, at other frequencies, the differences between the phases of the modified LPDA and the optimum solution were significant, particularly in the lower frequency range Antenna pattern are produced by modified LPDA and conventional LPDA at $2.04 \mathrm{Ghz}, 3.59 \mathrm{Ghz}$ and 4.5Ghz.
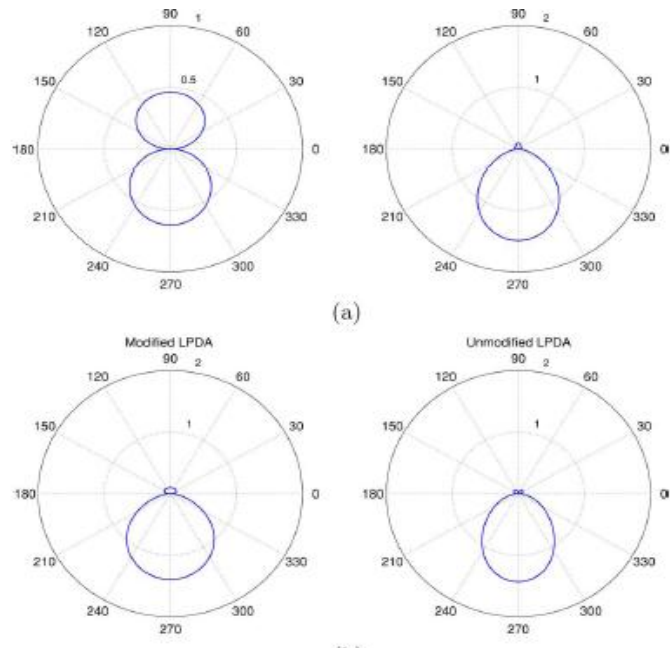

(a)
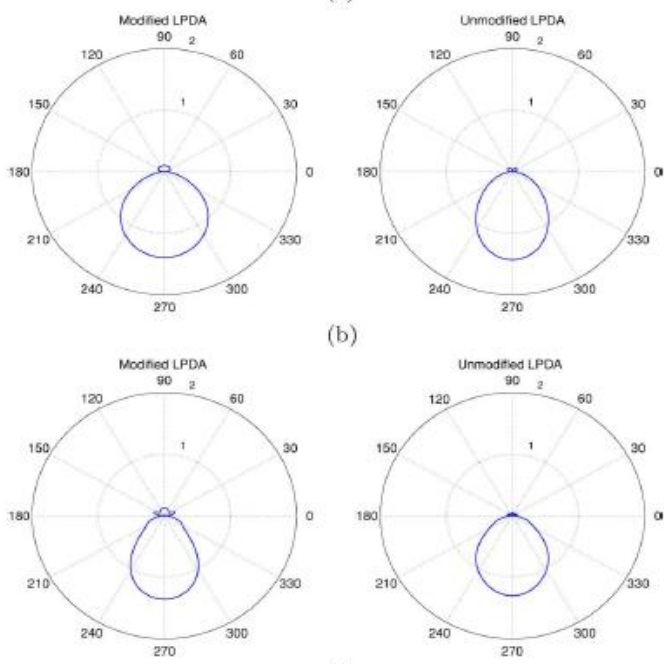

(c)

Fig.3. E plane antenna pattern produced by modified LPDA and unmodified LPDA

\section{CONCLUSION}

The HFSS simulations were performed for an eight element printed dipole LPDA antenna. Significant improvements in the output pulse fidelity were achieved for a dispersion engineered LPDA antenna with a large number of elements.

\section{REFERENCES}

[1] F. P. Casares-Miranda, C. Camacho-Penalosa, and C. Caloz, "High-gain active composite right/left-handed leaky-wave antenna," IEEE Trans. Antennas Propag., pp. 22292-2300, Aug. 2006.

[2] O. Siddiqui, M. Mojahedi, and G. Eleftheriades, "Periodically loaded transmission line with effective negative refractive index and negative group velocity,’IEEE Trans. Antenna Propag., pp. 2619-2625, Oct. 2003.

[3] A. Erentok and R. W. Ziolkowski, "A hybrid optimization method to analyze metamaterial-based electrically small antennas," IEEE Trans. Antennas and Propagation, pp. 731-741, Mar. 2007.

[4] F. P. Casares-Miranda, C. Camacho-Penalosa, and C. Caloz, "High-gain active composite right/left-handed leaky-wave antenna," IEEE Trans. Antennas Propag., pp. 2292-2300, Aug. 2006.

[5] S.-S. Oh and L. Shafai, "Compensated circuit with characteristics of lossless

[6] Double negative materials and its application to array atennas," IET Microw.Antenna Propag., Special Issue on Metamaterials LHM, pp. 29-38, Nov. 2007.

[7] D. Pozar, Microwave Engineering, 3rd ed. Hoboken, NJ: John Wiley \& Sons, 2004. 